TABLE 1. POINT-COUNTING PROPORTIONS OF SANDSTONES FROM THE ARPEROS, CUESTECITA, ESPERANZA, AND EL PAXTLE FORMATIONS

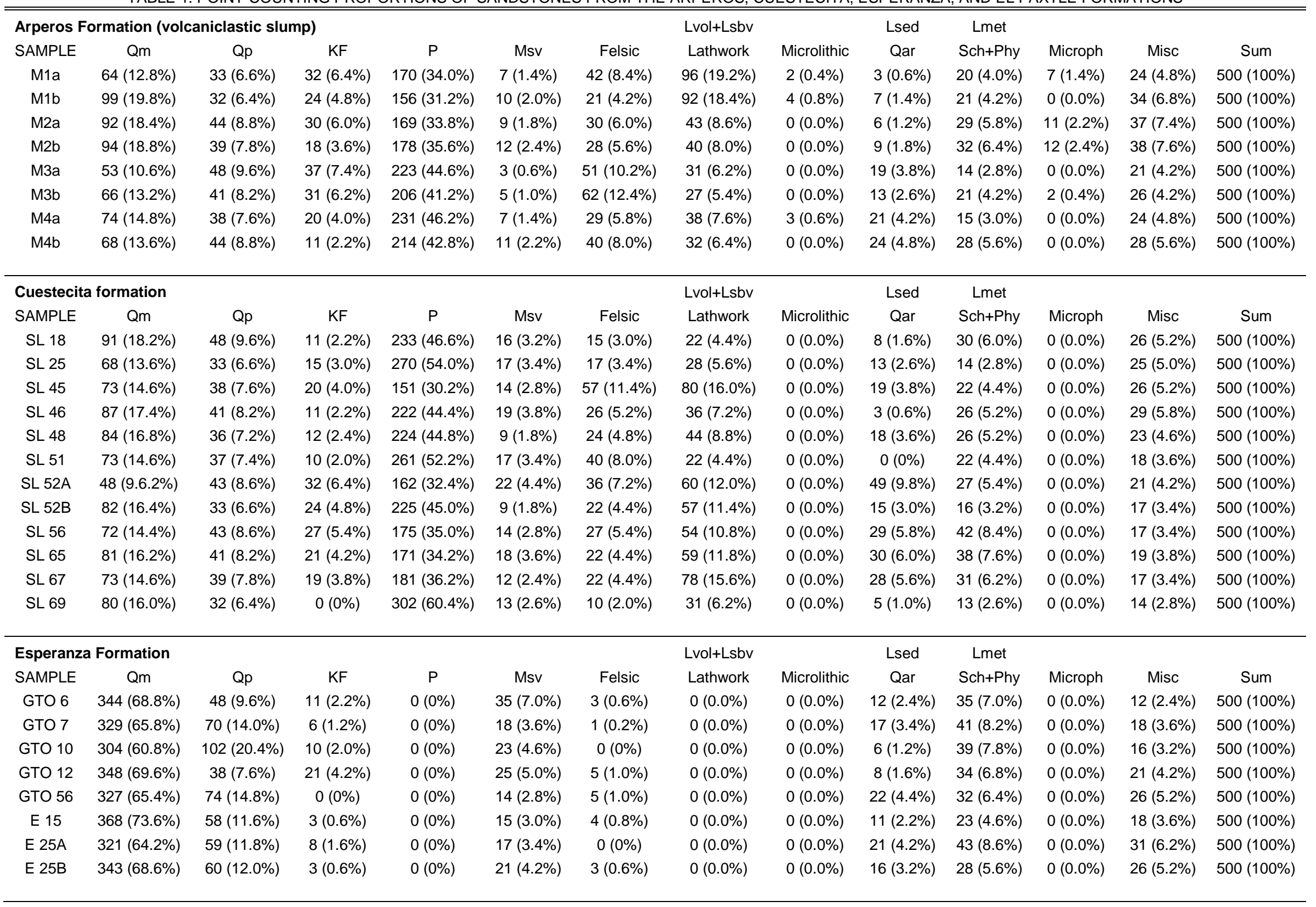




\begin{tabular}{|c|c|c|c|c|c|c|c|c|c|c|c|c|c|}
\hline \multicolumn{7}{|c|}{ EI Paxtle Formation } & \multicolumn{2}{|l|}{ Lvol+Lsbv } & \multirow{2}{*}{$\begin{array}{l}\text { Lsed } \\
\text { Qar }\end{array}$} & \multicolumn{2}{|l|}{ Lmet } & \multirow[b]{2}{*}{ Misc } & \multirow[b]{2}{*}{ Sum } \\
\hline SAMPLE & Qm & Qp & $\mathrm{KF}$ & $\mathrm{P}$ & Msv & Felsic & Lathwork & Microlithic & & Sch+Phy & Microph & & \\
\hline SL 21 & $19(3.8 \%)$ & $47(9.4 \%)$ & $0(0 \%)$ & $281(56.2 \%)$ & $0(0 \%)$ & $0(0.0 \%)$ & $129(25.8 \%)$ & $0(0.0 \%)$ & $0(0.0 \%)$ & $0(0.0 \%)$ & $0(0.0 \%)$ & $24(4.8 \%)$ & $500(100 \%)$ \\
\hline SL 22bis & $7(1.4 \%)$ & $53(10.6 \%)$ & $0(0 \%)$ & $218(43.6 \%)$ & $0(0 \%)$ & $0(0.0 \%)$ & $191(38.2 \%)$ & $0(0.0 \%)$ & $0(0.0 \%)$ & $0(0.0 \%)$ & $0(0.0 \%)$ & $31(6.2 \%)$ & $500(100 \%)$ \\
\hline SL 25A & $13(2.6 \%)$ & $64(12.8 \%)$ & $0(0 \%)$ & $196(39.2 \%)$ & $0(0 \%)$ & $0(0.0 \%)$ & $197(39.4 \%)$ & $0(0.0 \%)$ & $0(0.0 \%)$ & $0(0.0 \%)$ & $0(0.0 \%)$ & $30(6.0 \%)$ & $500(100 \%)$ \\
\hline SL 47 & $21(4.2 \%)$ & $53(10.6 \%)$ & $0(0 \%)$ & 267 (53.4\%) & $0(0 \%)$ & $0(0.0 \%)$ & $123(24.6 \%)$ & $0(0.0 \%)$ & $0(0.0 \%)$ & $0(0.0 \%)$ & $0(0.0 \%)$ & $36(7.2 \%)$ & $500(100 \%)$ \\
\hline
\end{tabular}

RECALCULATED PARAMETERS

\begin{tabular}{|c|c|c|c|c|c|c|c|c|c|}
\hline \multicolumn{10}{|c|}{ Arperos Formation (volcaniclastic slump) } \\
\hline SAMPLE & QmFL \%Qm & QmFL \%F & QmFL \%L & QtFL \%Qt & QtFL \%F & QtFL \%L & QpLvLm \%Qp & QpLvLm \%Lv & QpLvLm \%Lm \\
\hline M1a & 13.9 & 43.7 & 42.4 & 21.0 & 43.7 & 35.3 & 16.8 & 71.4 & 11.7 \\
\hline M1b & 21.7 & 39.5 & 38.8 & 28.7 & 39.5 & 31.8 & 18.1 & 66.1 & 15.8 \\
\hline $\mathrm{M} 2 \mathrm{a}$ & 20.8 & 44.9 & 34.3 & 30.7 & 44.9 & 24.4 & 28.9 & 48.0 & 23.0 \\
\hline $\mathrm{M} 2 \mathrm{~b}$ & 21.5 & 44.7 & 33.8 & 30.4 & 44.7 & 24.9 & 26.4 & 45.9 & 27.7 \\
\hline M3a & 11.1 & 54.6 & 34.2 & 21.2 & 54.6 & 24.2 & 29.4 & 50.3 & 20.2 \\
\hline M3b & 14.1 & 50.7 & 35.1 & 22.9 & 50.7 & 26.3 & 25.0 & 54.3 & 20.7 \\
\hline M4a & 15.8 & 53.5 & 30.7 & 23.9 & 53.5 & 22.6 & 26.4 & 48.6 & 25.0 \\
\hline M4b & 14.8 & 48.8 & 36.4 & 24.3 & 48.8 & 26.9 & 26.2 & 42.9 & 31.0 \\
\hline \multicolumn{10}{|c|}{ Cuestecita formation } \\
\hline SAMPLE & QmFL \%Qm & QmFL \%F & QmFL \%L & QtFL \%Qt & QtFL \%F & QtFL \%L & QpLvLm \%Qp & QpLvLm \%Lv & QpLvLm \%Lm \\
\hline SL 18 & 19.9 & 53.3 & 26.8 & 30.3 & 53.3 & 16.4 & 39.0 & 30.1 & 30.9 \\
\hline SL 25 & 14.8 & 62.2 & 23.0 & 22.0 & 62.2 & 15.8 & 31.4 & 42.8 & 25.8 \\
\hline SL 45 & 15.9 & 37.2 & 46.9 & 24.1 & 37.2 & 38.7 & 17.6 & 63.4 & 19.0 \\
\hline SL 46 & 19.2 & 51.5 & 29.3 & 28.3 & 51.5 & 20.2 & 31.1 & 47.0 & 21.9 \\
\hline SL 48 & 17.9 & 50.4 & 31.7 & 25.6 & 50.4 & 24.0 & 24.3 & 45.9 & 29.8 \\
\hline SL 51 & 15.7 & 58.3 & 26.0 & 23.6 & 58.3 & 18.1 & 30.6 & 51.2 & 18.2 \\
\hline SL 52A & 10.5 & 42.4 & 47.1 & 19.9 & 42.4 & 37.7 & 20.0 & 44.6 & 35.4 \\
\hline SL 52B & 17.3 & 52.5 & 41.6 & 24.3 & 52.5 & 23.2 & 23.1 & 55.2 & 21.7 \\
\hline SL 56 & 15.4 & 43.2 & 41.6 & 24.5 & 43.2 & 32.4 & 22.0 & 41.5 & 36.5 \\
\hline SL 65 & 17.5 & 41.5 & 41.0 & 26.3 & 41.5 & 32.2 & 21.6 & 42.6 & 35.8 \\
\hline SL 67 & 15.5 & 42.5 & 42.0 & 23.8 & 42.5 & 33.7 & 19.7 & 50.5 & 29.8 \\
\hline SL 69 & 16.9 & 63.8 & 19.3 & 23.7 & 63.8 & 12.5 & 35.2 & 45.0 & 19.8 \\
\hline
\end{tabular}




\begin{tabular}{|c|c|c|c|c|c|c|c|c|c|}
\hline \multicolumn{10}{|c|}{ Esperanza Formation } \\
\hline SAMPLE & QmFL \%Qm & QmFL \%F & QmFL \%L & QtFL \%Qt & QtFL \%F & QtFL \%L & QpLvLm \%Qp & QpLvLm \%Lv & QpLvLm \%Lm \\
\hline GTO 6 & 75.9 & 2.4 & 21.7 & 86.5 & 2.4 & 11.1 & 49.0 & 3.1 & 47.9 \\
\hline GTO 7 & 70.9 & 1.3 & 27.8 & 86.0 & 1.3 & 12.7 & 54.3 & 0.8 & 44.9 \\
\hline GTO 10 & 65.9 & 2.2 & 31.9 & 88.1 & 2.2 & 9.7 & 69.4 & 0.0 & 30.6 \\
\hline GTO 12 & 76.7 & 4.6 & 18.9 & 85.0 & 4.6 & 10.4 & 44.7 & 5.9 & 49.4 \\
\hline GTO 56 & 71.1 & 0.0 & 28.9 & 87.2 & 0.0 & 12.8 & 55.6 & 3.8 & 40.6 \\
\hline E 15 & 78.8 & 0.6 & 20.6 & 91.2 & 0.6 & 8.2 & 60.4 & 4.2 & 35.4 \\
\hline E 25A & 71.0 & 1.8 & 27.2 & 84.1 & 1.8 & 14.1 & 48.0 & 0.0 & 52.0 \\
\hline E 25B & 75.7 & 0.7 & 23.6 & 89.0 & 0.7 & 10.3 & 56.1 & 2.8 & 41.1 \\
\hline \multicolumn{10}{|c|}{ El Paxtle Formation } \\
\hline SAMPLE & QmFL \%Qm & QmFL \%F & QmFL \%L & QtFL \%Qt & QtFL \%F & QtFL \%L & QpLvLm \%Qp & QpLvLm \%Lv & QpLvLm \%Lm \\
\hline SL 21 & 4.0 & 59.0 & 37.0 & 13.9 & 59.0 & 27.1 & 26.7 & 73.3 & 0.0 \\
\hline SL 22bis & 1.5 & 46.5 & 52.0 & 12.8 & 46.5 & 40.7 & 21.7 & 78.3 & 0.0 \\
\hline SL 25A & 2.8 & 41.7 & 55.5 & 16.4 & 41.7 & 41.9 & 24.5 & 75.5 & 0.0 \\
\hline SL 47 & 4.5 & 57.5 & 37.9 & 15.9 & 57.5 & 26.6 & 30.1 & 69.9 & 0.0 \\
\hline
\end{tabular}

Point counting has been performed according to the Gazzi-Dickinson method (Dickinson, 1985). Values of the Cuestecita, Esperanza, and El Paxtle formations are from Martini et al. (2011) and are recalculated according to the new grain categories established for sandstones of the Arperos

Formation. Qm: monocrystalline quartz; Qp: polycrystalline quartz; KF: K-feldspar; P: plagioclase; Msv: muscovite; Lv + Lsbv: volcanic and subvolcanic grains; Lsed: sedimentary grain; Qar: quartz-rich very fine arenite and siltite; Lmet: metamorphic grain; Sch + Phy: schist and phyllites; Micoph: microphaneritic grain; Misc: cement, matrix, dense minerals, and post-depositional components.Qt: Qm + Qp; F: KF + P; Lm: Lsed + Lmet.

\section{REFERENCES CITED}

Dickinson, W.R., 1985, Interpreting provenance relations from detrital modes of sandstones, in Zuffa, G.G., ed., Provenance of arenites: Dordrecht, Netherlands, D. Reidel, NATO Advanced Study Institute Series, v. 148, p. 3-61.

Martini, M., Mori, L., Solari, L., and Centeno-García, E., 2011, Sandstone provenance of the Arperos Basin (Sierra de Guanajuato, central Mexico): Late Jurassic-Early Cretaceous back-arc spreading as the foundation of the Guerrero terrane: The Journal of Geology, v. 119, p. 597-617, doi:10.1086/661989. 
TABLE DR2.

SAMPLE M1a

(datum UTM nad 27: 2333786 N, 250650 E)

CORRECTED RATIOS

CORRECTED AGES (Ma)

\begin{tabular}{|c|c|c|c|c|c|c|c|c|c|c|c|c|c|c|c|c|c|}
\hline Analysis & $\mid \mathrm{U}(\mathrm{ppm})]^{\top}$ & Th (ppm) & $\mathrm{Th} / \mathrm{U}$ & ${ }^{207} \mathrm{~Pb} /{ }^{235} \mathrm{U}$ & $\pm 1 \mathrm{~s}$ & ${ }^{206} \mathrm{~Pb} /{ }^{238} \mathrm{U}$ & $\pm 1 \mathrm{~s}$ & Rho & ${ }^{206} \mathrm{~Pb} /{ }^{238} \mathrm{U}$ & $\pm 1 \mathrm{~s}$ & ${ }^{207} \mathrm{~Pb} /{ }^{235} \mathrm{U}$ & $\pm 1 \mathrm{~s}$ & ${ }^{207} \mathrm{~Pb} /{ }^{206} \mathrm{~Pb}$ & $\pm 1 \mathrm{~s}$ & Best age (Ma) & $\pm 1 \mathrm{~s}$ & $\%$ disc. \\
\hline Zircon $46 \quad 062$ & $\underline{515}$ & $\underline{284}$ & $\underline{0.52}$ & $\underline{0.02916}$ & $\underline{0.0014}$ & $\underline{0.00471}$ & $\underline{0.00006}$ & $\underline{0.26}$ & $\underline{30.3}$ & $\underline{0.4}$ & $\underline{29}$ & $\underline{1}$ & $\underline{-6}$ & $\underline{103}$ & $\underline{30}$ & $\underline{0}$ & $\underline{-4.5}$ \\
\hline Zircon $18 \quad 028$ & $\underline{804}$ & $\underline{325}$ & $\underline{0.38}$ & $\underline{0.03892}$ & $\underline{0.0017}$ & $\underline{0.00488}$ & $\underline{0.00005}$ & $\underline{0.26}$ & $\underline{31.4}$ & $\underline{0.3}$ & $\underline{39}$ & $\underline{2}$ & $\underline{563}$ & $\underline{86}$ & $\underline{31}$ & $\underline{0}$ & $\underline{19.5}$ \\
\hline Zircon $95 \quad 120$ & $\underline{550}$ & $\underline{237}$ & $\underline{\overline{0.41}}$ & $\underline{0.03489}$ & $\underline{0.0024}$ & $\underline{0.00489}$ & $\underline{0.00007}$ & $\underline{0.25}$ & $\underline{31.4}$ & $\overline{0.4}$ & $\underline{35}$ & $\underline{2}$ & $\underline{275}$ & $\underline{139}$ & $\underline{31}$ & $\underline{0}$ & $\underline{10.3}$ \\
\hline Zircon $08 \quad 016$ & $\underline{288}$ & $\underline{146}$ & $\underline{0.48}$ & $\underline{0.04547}$ & $\underline{0.0064}$ & $\underline{0.0049}$ & $\underline{0.0001}$ & $\underline{0.31}$ & $\underline{31.5}$ & $\underline{0.6}$ & $\underline{45}$ & $\underline{6}$ & $\underline{848}$ & $\underline{263}$ & $\underline{32}$ & $\underline{1}$ & $\underline{30.0}$ \\
\hline Zircon 77099 & $\underline{1108}$ & $\underline{488}$ & $\underline{0.41}$ & $\underline{0.03577}$ & $\underline{0.0019}$ & $\underline{0.005}$ & $\underline{0.00005}$ & $\underline{0.27}$ & $\underline{32.2}$ & $\underline{0.4}$ & $\underline{36}$ & $\underline{2}$ & $\underline{279}$ & $\underline{105}$ & $\underline{32}$ & $\underline{0}$ & $\underline{10.6}$ \\
\hline Zircon $11 \quad 020$ & $\underline{197}$ & $\underline{148}$ & $\underline{0.71}$ & $\underline{0.04741}$ & $\underline{0.005}$ & $\underline{0.00505}$ & $\underline{0.0001}$ & $\underline{0.29}$ & $\underline{32.5}$ & $\underline{0.7}$ & $\underline{47}$ & $\underline{5}$ & $\underline{871}$ & $\underline{189}$ & $\underline{33}$ & $\underline{1}$ & $\underline{30.9}$ \\
\hline Zircon $90 \quad 114$ & $\underline{294}$ & $\underline{119}$ & $\underline{0.38}$ & $\underline{0.04318}$ & $\underline{0.0035}$ & $\underline{0.00508}$ & 0.00009 & $\underline{0.24}$ & $\underline{32.7}$ & $\underline{0.6}$ & $\underline{43}$ & $\underline{3}$ & $\underline{660}$ & $\underline{158}$ & $\underline{33}$ & $\underline{1}$ & $\underline{24.0}$ \\
\hline Zircon $68 \quad 088$ & $\underline{991}$ & $\underline{384}$ & $\underline{0.37}$ & $\underline{0.04029}$ & $\underline{0.0043}$ & $\underline{0.00511}$ & $\underline{0.00007}$ & $\underline{0.27}$ & $\underline{32.8}$ & $\underline{0.4}$ & $\underline{40}$ & $\underline{4}$ & $\underline{501}$ & $\underline{215}$ & $\underline{33}$ & $\underline{0}$ & $\underline{18.0}$ \\
\hline Zircon $96 \quad 122$ & $\underline{1218}$ & $\underline{542}$ & $\underline{0.42}$ & $\underline{0.04265}$ & $\underline{0.0025}$ & $\underline{0.00537}$ & $\underline{0.00007}$ & $\underline{0.3}$ & $\underline{34.5}$ & $\underline{0.4}$ & $\underline{42}$ & $\underline{\mathbf{2}}$ & $\underline{513}$ & $\underline{113}$ & $\underline{35}$ & $\underline{0}$ & $\underline{17.9}$ \\
\hline Zircon $04 \quad 011$ & $\underline{50}$ & $\underline{24}$ & $\underline{0.46}$ & $\underline{0.07002}$ & $\underline{0.015}$ & 0.00584 & $\underline{0.00022}$ & $\underline{0.29}$ & $\underline{38}$ & $\underline{1}$ & $\underline{69}$ & $\underline{14}$ & $\underline{1359}$ & $\underline{383}$ & $\underline{38}$ & $\underline{1}$ & $\underline{44.9}$ \\
\hline Zircon_24_035 & $\underline{270}$ & $\underline{130}$ & $\underline{0.46}$ & $\underline{0.12758}$ & $\underline{0.0096}$ & $\underline{0.01758}$ & $\underline{0.00022}$ & $\underline{0.19}$ & $\underline{112}$ & $\underline{1}$ & $\underline{122}$ & $\underline{9}$ & $\underline{312}$ & $\underline{167}$ & $\underline{112}$ & $\underline{1}$ & $\underline{8.2}$ \\
\hline Zircon_31_044 & 746 & $\overline{994}$ & 1.26 & 0.13272 & 0.0028 & 0.01796 & 0.00012 & $\overline{0.32}$ & 114.8 & $0 . \overline{8}$ & $\overline{127}$ & 3 & $\overline{354}$ & 47 & 115 & $\overline{1}$ & 9.6 \\
\hline Zircon $09 \quad 017$ & $\underline{339}$ & $\underline{221}$ & $\underline{0.61}$ & $\underline{0.13614}$ & $\underline{0.0115}$ & $\underline{0.01825}$ & $\underline{0.00023}$ & $\underline{0.22}$ & $\underline{117}$ & $\underline{1}$ & $\underline{130}$ & $\underline{10}$ & $\underline{376}$ & $\underline{165}$ & $\underline{117}$ & $\underline{1}$ & $\underline{10.0}$ \\
\hline Zircon_97_123 & 600 & 254 & 0.40 & 0.13196 & 0.0032 & 0.01853 & 0.00013 & 0.3 & 118.4 & 0.8 & $\overline{126}$ & 3 & $\overline{279}$ & 50 & $\overline{118}$ & 1 & 6.0 \\
\hline Zircon_70_090 & 376 & 267 & 0.67 & 0.13416 & 0.0065 & 0.01866 & 0.00018 & 0.31 & 119 & 1 & 128 & 6 & 292 & 94 & 119 & 1 & 7.0 \\
\hline Zircon_58_076 & 267 & 76 & 0.27 & 0.14351 & 0.0081 & 0.01865 & 0.00017 & 0.21 & 119 & 1 & 136 & 7 & 445 & 125 & 119 & 1 & 12.5 \\
\hline Zircon_15_024 & 169 & 102 & 0.57 & 0.15017 & 0.0077 & 0.01892 & 0.00023 & 0.23 & 121 & 1 & 142 & 7 & 549 & 103 & 121 & 1 & 14.8 \\
\hline Zircon_33_046 & 486 & 465 & 0.90 & 0.13904 & 0.0036 & 0.01904 & 0.00015 & 0.3 & 121.6 & 0.9 & 132 & 3 & 321 & 59 & 122 & 1 & 7.9 \\
\hline Zircon_69_089 & 269 & 233 & 0.82 & 0.14274 & 0.0051 & 0.01903 & 0.00025 & 0.37 & 122 & 2 & 135 & 4 & 396 & 70 & 122 & 2 & 9.6 \\
\hline Zircon_79_101 & 193 & 111 & 0.54 & 0.15001 & 0.006 & 0.01915 & 0.00019 & 0.24 & 122 & 1 & 142 & 5 & 516 & 81 & 122 & 1 & 14.1 \\
\hline Zircon $17 \quad 027$ & $\underline{140}$ & $\underline{68}$ & $\underline{0.46}$ & $\underline{0.15486}$ & $\underline{0.0145}$ & $\underline{0.01922}$ & $\underline{0.00028}$ & $\underline{0.35}$ & $\underline{123}$ & $\underline{2}$ & $\underline{146}$ & $\underline{13}$ & $\underline{546}$ & $\underline{177}$ & $\underline{123}$ & $\underline{2}$ & $\underline{15.8}$ \\
\hline Zircon_64_083 & 543 & 467 & 0.81 & 0.14413 & 0.0031 & 0.01948 & 0.00016 & 0.38 & 124 & 1 & 137 & 3 & 359 & 47 & 124 & 1 & 9.5 \\
\hline Zircon $26 \quad 038$ & $\underline{84}$ & $\underline{44}$ & $\underline{0.49}$ & $\underline{0.15761}$ & $\underline{0.0135}$ & $\underline{0.01946}$ & $\underline{0.0003}$ & $\underline{0.24}$ & $\underline{124}$ & $\underline{2}$ & $\underline{149}$ & $\underline{12}$ & $\underline{558}$ & $\underline{183}$ & $\underline{124}$ & $\underline{\mathbf{2}}$ & $\underline{16.8}$ \\
\hline$\overline{\text { Zircon_78_100 }}$ & $5 \overline{43}$ & $2 \overline{40}$ & $\overline{0.42}$ & 0.1326 & $\overline{0.0037}$ & $\overline{0.01946}$ & $0 . \overline{00013}$ & $\overline{0.25}$ & $12 \overline{4.2}$ & $0 . \overline{8}$ & $\overline{126}$ & $\overline{3}$ & $\overline{174}$ & $\overline{60}$ & $\overline{124}$ & $\overline{1}$ & $\overline{1.4}$ \\
\hline Zircon_21_032 & 181 & 101 & 0.52 & 0.15528 & 0.0073 & 0.0196 & 0.00022 & 0.23 & 125 & 1 & 147 & 6 & 524 & 105 & 125 & 1 & 15.0 \\
\hline Zircon_12_021 & 211 & 55 & 0.25 & 0.15784 & 0.0061 & 0.01969 & 0.00022 & 0.28 & 126 & 1 & 149 & 5 & 543 & 76 & 126 & 1 & 15.4 \\
\hline Zircon_54_071 & 120 & 74 & 0.58 & 0.16763 & 0.0081 & 0.0197 & 0.00024 & 0.24 & 126 & 2 & 157 & 7 & 692 & 105 & 126 & 2 & 19.7 \\
\hline Zircon_38_052 & 188 & 103 & 0.51 & 0.12827 & 0.0088 & 0.01968 & 0.00026 & 0.27 & 126 & 2 & 123 & 8 & 62 & 140 & 126 & 2 & -2.4 \\
\hline Zircon $91 \quad 116$ & $\underline{137}$ & $\underline{71}$ & $\underline{0.49}$ & $\underline{0.16778}$ & $\underline{0.0111}$ & $\underline{0.01977}$ & $\underline{0.00029}$ & $\underline{0.32}$ & $\underline{126}$ & $\underline{2}$ & $\underline{157}$ & $\underline{10}$ & $\underline{659}$ & $\underline{122}$ & $\underline{126}$ & $\underline{\mathbf{2}}$ & $\underline{19.7}$ \\
\hline Zircon $93 \quad 118$ & $\underline{141}$ & $\underline{78}$ & $\underline{0.52}$ & $\underline{0.1606}$ & $\underline{0.012}$ & $\underline{0.01976}$ & $\underline{0.00025}$ & $\underline{0.26}$ & $\underline{126}$ & $\underline{2}$ & $\underline{151}$ & $\underline{11}$ & $\underline{565}$ & $\underline{147}$ & $\underline{126}$ & $\underline{\mathbf{2}}$ & $\underline{16.6}$ \\
\hline Zircon_34_047 & 411 & 272 & 0.62 & 0.13412 & 0.0036 & 0.01982 & 0.00017 & 0.32 & 127 & 1 & 128 & 3 & 151 & 61 & 127 & 1 & 0.8 \\
\hline Zircon_41_056 & 873 & 451 & 0.49 & 0.14257 & 0.0037 & 0.01986 & 0.0002 & 0.39 & 127 & 1 & 135 & 3 & 291 & 57 & 127 & 1 & 5.9 \\
\hline Zircon_59_077 & 245 & 149 & 0.57 & 0.15104 & 0.0056 & 0.01987 & 0.00019 & 0.27 & 127 & 1 & 143 & 5 & 436 & 84 & 127 & 1 & 11.2 \\
\hline Zircon_60_078 & 441 & 310 & 0.66 & 0.14814 & 0.0042 & 0.02029 & 0.00016 & 0.29 & 129 & 1 & 140 & 4 & 329 & 64 & 129 & 1 & 7.9 \\
\hline Zircon_43_058 & 207 & 144 & 0.66 & 0.15987 & 0.0062 & 0.02019 & 0.00024 & 0.31 & 129 & 2 & 151 & 5 & 515 & 85 & 129 & 2 & 14.6 \\
\hline Zircon_35_048 & 503 & 484 & 0.91 & 0.15505 & 0.0042 & 0.02027 & 0.00015 & 0.26 & 129.4 & 0.9 & 146 & 4 & 438 & 60 & 129 & 1 & 11.4 \\
\hline Zircon_44_059 & 298 & 199 & 0.63 & 0.15406 & 0.0047 & 0.02039 & 0.00018 & 0.29 & 130 & 1 & 145 & 4 & 398 & 67 & 130 & 1 & 10.3 \\
\hline Zircon_14_023 & 234 & 139 & 0.56 & 0.1671 & 0.0062 & 0.0206 & 0.00021 & 0.27 & 131 & 1 & 157 & 5 & 568 & 73 & 131 & 1 & 16.6 \\
\hline Zircon_13_022 & 468 & 478 & 0.96 & 0.15224 & 0.004 & 0.02073 & 0.00016 & 0.28 & 132 & 1 & 144 & 4 & 348 & 53 & 132 & 1 & 8.3 \\
\hline Zircon_66_086 & 253 & 177 & 0.66 & 0.15363 & 0.0065 & 0.02072 & 0.00019 & 0.22 & 132 & 1 & 145 & 6 & 357 & 97 & 132 & 1 & 9.0 \\
\hline Zircon $40 \quad 054$ & $\underline{133}$ & 101 & $\underline{0.72}$ & $\underline{0.16392}$ & $\underline{0.0121}$ & $\underline{0.02074}$ & $\underline{0.00031}$ & $\underline{0.26}$ & 132 & $\underline{2}$ & $\underline{154}$ & $\underline{11}$ & $\underline{504}$ & $\underline{154}$ & $\underline{132}$ & $\underline{2}$ & $\underline{14.3}$ \\
\hline Zircon_32_045 & 411 & 249 & 0.57 & 0.14601 & 0.004 & 0.02079 & 0.00015 & 0.29 & 132.6 & 0.9 & 138 & 3 & 238 & 62 & 133 & 1 & 3.9 \\
\hline Zircon_42_057 & 105 & 59 & 0.53 & 0.15918 & 0.0086 & 0.02083 & 0.00023 & 0.2 & 133 & 1 & 150 & 8 & 428 & 124 & 133 & 1 & 11.3 \\
\hline Zircon_56_074 & 629 & 686 & 1.03 & 0.14436 & 0.0032 & 0.02108 & 0.00016 & 0.33 & 134 & 1 & 137 & 3 & 184 & 51 & 134 & 1 & 2.2 \\
\hline Zircon_39_053 & 164 & 112 & 0.64 & 0.15519 & 0.0056 & 0.02098 & 0.00017 & 0.24 & 134 & 1 & 146 & 5 & 363 & 82 & 134 & 1 & 8.2 \\
\hline Zircon_86_110 & 341 & 126 & 0.35 & 0.14914 & 0.0082 & 0.02114 & 0.0002 & 0.22 & 135 & 1 & 141 & 7 & 249 & 112 & 135 & 1 & 4.3 \\
\hline Zircon $48 \quad 064$ & $\underline{418}$ & $\underline{132}$ & $\underline{0.30}$ & $\underline{0.16882}$ & $\underline{0.0156}$ & $\underline{0.02124}$ & $\underline{0.00037}$ & $\underline{0.36}$ & $\underline{135}$ & $\underline{2}$ & $\underline{158}$ & $\underline{14}$ & $\underline{517}$ & $\underline{198}$ & $\underline{135}$ & $\underline{2}$ & $\underline{14.6}$ \\
\hline Zircon_28_040 & 397 & 292 & 0.69 & 0.15339 & 0.0039 & 0.02134 & 0.00019 & 0.35 & 136 & 1 & 145 & 3 & 303 & 57 & 136 & 1 & 6.2 \\
\hline Zircon_83_106 & 265 & 234 & 0.83 & 0.17287 & 0.0068 & 0.02131 & 0.0002 & 0.24 & 136 & 1 & 162 & 6 & 573 & 79 & 136 & 1 & 16.0 \\
\hline Zircon_81_104 & 138 & 72 & 0.49 & 0.16872 & 0.0077 & 0.02135 & 0.00023 & 0.24 & 136 & 1 & 158 & 7 & 500 & 92 & 136 & 1 & 13.9 \\
\hline Zircon_55_072 & 459 & 252 & 0.52 & 0.14937 & 0.0036 & 0.02142 & 0.00016 & 0.32 & 137 & 1 & 141 & 3 & 222 & 55 & 137 & 1 & 2.8 \\
\hline Zircon_20_030 & 317 & 155 & 0.46 & 0.16719 & 0.005 & 0.02143 & 0.00017 & 0.27 & 137 & 1 & 157 & 4 & 475 & 67 & 137 & 1 & 12.7 \\
\hline Zircon_01_Ma1_ & 246 & 262 & 1.00 & 0.1611 & 0.0062 & 0.02187 & 0.00028 & 0.34 & 139 & 2 & 152 & 5 & 369 & 76 & 139 & 2 & 8.6 \\
\hline Zircon $84 \quad 107$ & $\underline{149}$ & $\underline{87}$ & $\underline{0.55}$ & $\underline{0.16146}$ & $\underline{0.0092}$ & $\underline{0.02177}$ & $\underline{0.00025}$ & $\underline{0.25}$ & $\underline{139}$ & $\underline{2}$ & $\underline{152}$ & $\underline{8}$ & $\underline{362}$ & $\underline{112}$ & $\underline{139}$ & $\underline{2}$ & $\underline{8.6}$ \\
\hline Zircon_76_098 & 168 & 112 & 0.63 & 0.17311 & 0.0064 & 0.02216 & 0.00021 & 0.25 & 141 & 1 & 162 & 6 & 490 & 75 & 141 & 1 & 13.0 \\
\hline Zircon_29_041 & 359 & 224 & 0.59 & 0.16641 & 0.0086 & 0.02206 & 0.00027 & 0.32 & 141 & 2 & 156 & 7 & 400 & 106 & 141 & 2 & 9.6 \\
\hline Zircon_72_093 & 404 & 298 & 0.70 & 0.17495 & 0.0048 & 0.02231 & 0.00019 & 0.31 & 142 & 1 & 164 & 4 & 497 & 54 & 142 & 1 & 13.4 \\
\hline Zircon_50_066 & 296 & 150 & 0.48 & 0.16705 & 0.0052 & 0.02234 & 0.00016 & 0.25 & 142 & 1 & 157 & 4 & 391 & 70 & 142 & 1 & 9.6 \\
\hline Zircon_49_065 & 244 & 213 & 0.82 & 0.17241 & 0.0063 & 0.0223 & 0.00022 & 0.27 & 142 & 1 & 162 & 5 & 462 & 81 & 142 & 1 & 12.3 \\
\hline Zircon $71 \quad 092$ & $\underline{536}$ & $\underline{281}$ & $\underline{0.49}$ & $\underline{0.1519}$ & $\underline{0.0093}$ & $\underline{0.0222}$ & $\underline{0.00023}$ & $\underline{0.29}$ & $\underline{142}$ & $\underline{1}$ & $\underline{144}$ & $\underline{8}$ & $\underline{177}$ & $\underline{120}$ & $\underline{142}$ & $\underline{1}$ & $\underline{1.4}$ \\
\hline Zircon_92_117 & 205 & 70 & 0.32 & 0.15806 & 0.0068 & 0.02276 & 0.00021 & 0.21 & $\overline{145}$ & 1 & 149 & 6 & 226 & 92 & 145 & 1 & 2.7 \\
\hline Zircon_27_039 & 277 & 153 & 0.52 & 0.15791 & 0.0057 & 0.02285 & 0.00037 & 0.45 & 146 & 2 & 149 & 5 & 202 & 77 & 146 & 2 & 2.0 \\
\hline Zircon_57_075 & 129 & 73 & 0.54 & 0.16529 & 0.0081 & 0.02294 & 0.00025 & 0.22 & 146 & 2 & 155 & 7 & 303 & 115 & 146 & 2 & 5.8 \\
\hline Zircon 22033 & $\underline{235}$ & $\underline{119}$ & $\underline{0.48}$ & $\underline{0.17174}$ & $\underline{0.0112}$ & $\underline{0.02288}$ & $\underline{0.00023}$ & $\underline{0.21}$ & $\underline{146}$ & $\underline{1}$ & $\underline{161}$ & $\underline{10}$ & $\underline{389}$ & $\underline{144}$ & $\underline{146}$ & $\underline{1}$ & $\underline{9.3}$ \\
\hline Zircon_37_051 & 121 & 92 & 0.72 & 0.17418 & 0.0087 & 0.02324 & 0.0004 & 0.34 & 148 & 3 & 163 & 8 & 409 & 110 & 148 & 3 & 9.2 \\
\hline Zircon_07_015 & 984 & 983 & 0.94 & 0.16723 & 0.0037 & 0.02345 & 0.00018 & 0.33 & 149 & 1 & 157 & 3 & 278 & 45 & 149 & 1 & 5.1 \\
\hline Zircon_87_111 & 439 & 205 & 0.44 & 0.18451 & 0.0072 & 0.02388 & 0.00021 & 0.31 & 152 & 1 & 172 & 6 & 454 & 75 & 152 & 1 & 11.6 \\
\hline Zircon_06_014 & 432 & 281 & 0.61 & 0.16515 & 0.0038 & 0.02432 & 0.00016 & 0.28 & 155 & 1 & 155 & 3 & 171 & 48 & 155 & 1 & 0.0 \\
\hline Zircon_80_102 & 331 & 69 & 0.20 & 0.17857 & 0.0052 & 0.02427 & 0.0002 & 0.27 & 155 & 1 & 167 & 4 & 349 & 60 & 155 & 1 & 7.2 \\
\hline Zircon $03 \quad 010$ & $\underline{369}$ & $\underline{239}$ & $\underline{0.61}$ & $\underline{0.18226}$ & $\underline{0.0223}$ & $\underline{0.02486}$ & $\underline{0.00055}$ & $\underline{0.35}$ & $\underline{158}$ & $\underline{3}$ & $\underline{170}$ & $\underline{19}$ & $\underline{336}$ & $\underline{230}$ & $\underline{158}$ & $\underline{3}$ & $\underline{7.1}$ \\
\hline
\end{tabular}




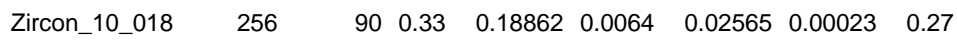

Zircon_02_009

Zircon 30042

Zircon_85_108

Zircon $53 \quad 070$

Zircon 74095

Zircon $94 \quad 119$

Zircon_05_012

Zircon_82_105

Zircon_75_096

Zircon_23_034

Zircon_67_087

Zircon_45_060

Zircon_36_050

Zircon 62081

Zircon_63_082

Zircon_61_080

Zircon 88

Zircon_89_113

Zircon_99_125

Zircon_98_124

Zircon_51_068

Zircon_100_126

Zircon_25_036

Zircon 65084

Zircon_16_026

Zircon_52_069

Zircon 19029

Zircon_73_094

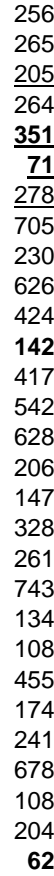

$\begin{array}{llllllll}117 & 0.42 & 0.18633 & 0.0073 & 0.0256 & 0.00023 & 0.24\end{array}$

$\begin{array}{lllllll}108 & \underline{0.50} & \underline{0.20545} & \underline{0.0113} & \underline{0.02655} & \underline{0.00032} & \underline{0.29}\end{array}$

$\begin{array}{llllllll}47 & 0.17 & 0.22751 & 0.0113 & 0.02655 & 0.0032 & 0.29\end{array}$

$\begin{array}{lllllll}152 & 0.41 & \underline{0.27276} & \underline{0.0176} & \underline{0.0287} & \underline{0.00169} & \underline{0.92}\end{array}$

$\begin{array}{lllllllll}34 & 0.45 & 0.2746 & 0.0337 & 0.02953 & 0.00129 & 0.64\end{array}$

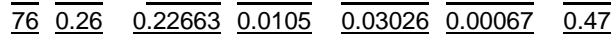

$\begin{array}{lllllll}698 & \frac{0.26}{0.93} & 0.24399 & 0.0055 & 0.03026 & 0.000041 & 0.47\end{array}$

$\begin{array}{llllllll}159 & 0.65 & 0.24636 & 0.0073 & 0.03438 & 0.00041 & 0.41\end{array}$

$\begin{array}{lllllll}125 & 0.19 & 0.27028 & 0.0071 & 0.0365 & 0.00062 & 0.64\end{array}$

$\begin{array}{lllllll}187 & 0.41 & 0.36693 & 0.0072 & 0.04862 & 0.00037 & 0.39\end{array}$

$\begin{array}{llllllll}51 & 0.34 & 0.49603 & 0.0215 & 0.0514 & 0.00089 & 0.51\end{array}$

$\begin{array}{llllllll}344 & 0.78 & 0.42903 & 0.008 & 0.05738 & 0.00044 & 0.41\end{array}$

$\begin{array}{lllllll}270 & 0.47 & 0.63808 & 0.0102 & 0.07952 & 0.00045 & 0.37\end{array}$

$\begin{array}{llllllll}271 & 0.41 & 0.66035 & 0.012 & 0.08023 & 0.00051 & 0.34\end{array}$

$\begin{array}{llllllll}211 & 0.96 & 0.62249 & 0.0147 & 0.08141 & 0.0007 & 0.36\end{array}$

$\begin{array}{rllllll}75 & 0.48 & 0.76584 & 0.0177 & 0.08659 & 0.00082 & 0.41\end{array}$

$\begin{array}{llllllll}161 & 0.46 & 0.87756 & 0.0154 & 0.10338 & 0.00075 & 0.42\end{array}$

$\begin{array}{llllllll}109 & 0.39 & 0.91602 & 0.0164 & 0.10663 & 0.00086 & 0.44\end{array}$

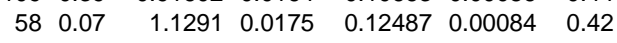

$\begin{array}{lllllll}87 & 0.61 & 1.1489 & 0.0259 & 0.1262 & 0.00102 & 0.37\end{array}$

$\begin{array}{llllllll}153 & 1.34 & 1.39589 & 0.0765 & 0.14481 & 0.00155 & 0.42\end{array}$

$\begin{array}{lllllll}142 & 0.29 & 1.6802 & 0.0254 & 0.16604 & 0.00095 & 0.37\end{array}$

$\begin{array}{llllllll}64 & 0.35 & 1.9544 & 0.033 & 0.18277 & 0.00143 & 0.47\end{array}$

$\begin{array}{llllllll}147 & 0.57 & 2.3769 & 0.0397 & 0.21033 & 0.00191 & 0.54\end{array}$

$\begin{array}{lllllll}168 & 0.23 & 2.2097 & 0.0318 & 0.19439 & 0.00121 & 0.42\end{array}$

$\begin{array}{llllllll}145 & 1.27 & 3.0249 & 0.0562 & 0.24081 & 0.00181 & 0.4\end{array}$

$\begin{array}{lllllll}115 & 0.53 & 4.2191 & 0.0613 & 0.28421 & 0.00185 & 0.45\end{array}$

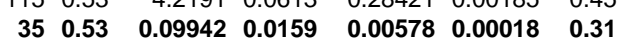

\begin{tabular}{|c|c|c|c|c|c|c|c|c|}
\hline 163 & 1 & 175 & 6 & 348 & 70 & 163 & 1 & 6.9 \\
\hline 163 & 1 & 173 & 6 & 340 & 80 & 163 & 1 & 5.8 \\
\hline 169 & $\underline{2}$ & $\underline{190}$ & $\underline{10}$ & 457 & $\underline{115}$ & $\underline{169}$ & $\underline{2}$ & 11.1 \\
\hline$\overline{180}$ & $\overline{1}$ & $\overline{208}$ & $\overline{6}$ & $\overline{545}$ & 68 & $\overline{180}$ & $\overline{1}$ & $\overline{13.5}$ \\
\hline 182 & $\underline{11}$ & $\underline{245}$ & $\underline{14}$ & $\underline{786}$ & $\underline{57}$ & $\underline{182}$ & $\underline{11}$ & $\underline{25.7}$ \\
\hline$\underline{188}$ & $\underline{8}$ & 246 & $\underline{27}$ & 852 & $\underline{191}$ & $\underline{188}$ & $\underline{8}$ & 23.6 \\
\hline$\underline{192}$ & $\underline{4}$ & $\underline{207}$ & $\underline{9}$ & $\overline{408}$ & $\underline{87}$ & $\overline{192}$ & $\underline{4}$ & $\underline{7.2}$ \\
\hline 216 & $\overline{3}$ & $\overline{222}$ & $\overline{4}$ & $\overline{281}$ & $\overline{41}$ & $\overline{216}$ & $\overline{3}$ & $\overline{2.7}$ \\
\hline 218 & 3 & 224 & 6 & 285 & 58 & 218 & 3 & 2.7 \\
\hline 231 & 4 & 243 & 6 & 362 & 43 & 231 & 4 & 4.9 \\
\hline 306 & 2 & 317 & 5 & 407 & 42 & 306 & 2 & 3.5 \\
\hline 323 & 5 & 409 & 15 & 928 & 74 & 323 & 5 & 21.0 \\
\hline 360 & 3 & 362 & 6 & 379 & 40 & 360 & 3 & 0.6 \\
\hline 493 & 3 & 501 & 6 & 539 & 34 & 493 & 3 & 1.6 \\
\hline 497 & 3 & 515 & 7 & 595 & 38 & 497 & 3 & 3.5 \\
\hline 505 & 4 & 491 & 9 & 445 & 51 & 505 & 4 & -2.9 \\
\hline 535 & 5 & 577 & 10 & 747 & 46 & 535 & 5 & 7.3 \\
\hline 634 & 4 & 640 & 8 & 659 & 32 & 634 & 4 & 0.9 \\
\hline 653 & 5 & 660 & 9 & 683 & 32 & 653 & 5 & 1.1 \\
\hline 759 & 5 & 767 & 8 & 790 & 28 & 759 & 5 & 1.0 \\
\hline 766 & 6 & 777 & 12 & 802 & 42 & 766 & 6 & 1.4 \\
\hline 872 & 9 & 887 & 32 & 926 & 92 & 872 & 9 & 1.7 \\
\hline 990 & 5 & 1001 & 10 & 1022 & 27 & 1022 & 27 & 1.1 \\
\hline 1082 & 8 & 1100 & 11 & 1136 & 31 & 1136 & 31 & 1.6 \\
\hline 1231 & 10 & 1236 & 12 & 1243 & 29 & 1243 & 29 & 0.4 \\
\hline 1145 & 7 & 1184 & 10 & 1244 & 24 & 1244 & 24 & 3.3 \\
\hline 1391 & 9 & 1414 & 14 & 1450 & 31 & 1450 & 31 & 1.6 \\
\hline 1613 & 9 & 1678 & 12 & 1761 & 25 & 1761 & 25 & 30 \\
\hline 37 & 1 & 96 & 15 & 2024 & 258 & 2024 & 258 & 61.5 \\
\hline
\end{tabular}

SAMPLE M2a

(datum UTM nad 27: $2333786 \mathrm{~N}, 250650 \mathrm{E}$ )

CORRECTED RATIOS

CORRECTED AGES (Ma)

\begin{tabular}{|c|c|c|c|c|c|c|c|c|c|c|c|c|c|c|c|c|c|}
\hline Analysis & U (ppm) & Th (ppm) & $\mathrm{Th} / \mathrm{U}$ & ${ }^{207} \mathrm{~Pb} /{ }^{235} \mathrm{U}$ & $\pm 1 \mathrm{~s}$ & ${ }^{206} \mathrm{~Pb} /{ }^{238} \mathrm{U}$ & $\pm 1 \mathrm{~s}$ & Rho & ${ }^{206} \mathrm{~Pb} /{ }^{238} \mathrm{U}$ & $\pm 1 \mathrm{~s}$ & ${ }^{207} \mathrm{~Pb} /{ }^{235} \mathrm{U}$ & $\pm 1 \mathrm{~s}$ & ${ }^{207} \mathrm{~Pb} /{ }^{206} \mathrm{~Pb}$ & $\pm 1 \mathrm{~s}$ & Best age (Ma) & $\pm 1 \mathrm{~s}$ & \% disc. \\
\hline Zircon $39 \quad 053$ & 450 & 229 & 0.49 & 0.05209 & 0.011 & 0.00502 & 0.00014 & $\underline{0.3}$ & 32.3 & $\underline{0.9}$ & $\underline{52}$ & $\underline{11}$ & 1074 & $\underline{413}$ & 32 & $\underline{1}$ & 37.9 \\
\hline$\overline{\text { Zircon_12_021 }}$ & $\overline{294}$ & $\overline{207}$ & $\overline{0.67}$ & $\overline{0.04084}$ & $0 . \overline{0045}$ & $\overline{0.00503}$ & $\overline{0.00009}$ & $0 . \overline{19}$ & $\overline{32.4}$ & 0.6 & 41 & $\overline{4}$ & 563 & $\overline{208}$ & 32 & $\overline{1}$ & $\overline{21.0}$ \\
\hline Zircon_41_056 & 295 & 177 & 0.57 & 0.05147 & 0.0049 & 0.00514 & 0.00007 & 0.15 & 33.1 & 0.5 & 51 & 5 & 1003 & 187 & 33 & 1 & 35.1 \\
\hline Zircon_23_034 & 355 & 126 & 0.34 & 0.05401 & 0.0046 & 0.0056 & 0.00009 & 0.27 & 36 & 0.6 & 53 & 4 & 926 & 162 & 36 & 1 & 32.1 \\
\hline Zircon $14 \quad 023$ & $\underline{62}$ & 36 & 0.56 & 0.07116 & 0.0111 & 0.00658 & 0.00021 & $\underline{0.23}$ & 42 & 1 & 70 & 11 & 1158 & 270 & 42 & 1 & 40.0 \\
\hline Zircon_26_038 & 286 & $1 \overline{78}$ & $\overline{0.59}$ & 0.14237 & 0.0045 & $\overline{0.01858}$ & $\overline{0.00018}$ & 0.3 & $1 \overline{19}$ & $\overline{1}$ & 135 & 4 & 461 & 65 & $1 \overline{19}$ & $\overline{1}$ & $\overline{11.9}$ \\
\hline Zircon $03 \quad 010$ & $\underline{150}$ & 75 & 0.48 & 0.15081 & 0.0111 & $\underline{0.01864}$ & $\underline{0.00023}$ & $\underline{0.24}$ & 119 & $\underline{1}$ & 143 & 10 & $\underline{556}$ & 138 & 119 & $\underline{1}$ & 16.8 \\
\hline 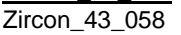 & $\overline{806}$ & $9 \overline{85}$ & $\overline{1.17}$ & $\overline{0.12755}$ & $\overline{0.0036}$ & $\overline{0.01885}$ & $\overline{0.00015}$ & $\overline{0.29}$ & $12 \overline{20.4}$ & $0 . \overline{9}$ & $\overline{122}$ & 3 & $\overline{163}$ & $\overline{62}$ & $\overline{120}$ & $\overline{1}$ & 1.3 \\
\hline Zircon_38_052 & 556 & 405 & 0.69 & 0.14164 & 0.0039 & 0.01894 & 0.00017 & 0.32 & 121 & 1 & 135 & 3 & 390 & 57 & 121 & 1 & 10.4 \\
\hline Zircon_25_036 & 1314 & 734 & 0.53 & 0.13971 & 0.009 & 0.0191 & 0.00018 & 0.19 & 122 & 1 & 133 & 8 & 331 & 135 & 122 & 1 & 8.3 \\
\hline Zircon_70_090 & 695 & 503 & 0.69 & 0.13966 & 0.0038 & 0.01955 & 0.00013 & 0.26 & 124.8 & 0.8 & 133 & 3 & 299 & 56 & 125 & 1 & 6.2 \\
\hline Zircon_10_018 & 235 & 187 & 0.76 & 0.15539 & 0.0053 & 0.01957 & 0.00019 & 0.28 & 125 & 1 & 147 & 5 & 523 & 68 & 125 & 1 & 15.0 \\
\hline Zircon_36_050 & 197 & 114 & 0.55 & 0.16151 & 0.0079 & 0.01961 & 0.00017 & 0.18 & 125 & 1 & 152 & 7 & 612 & 102 & 125 & 1 & 17.8 \\
\hline Zircon_72_093 & 882 & 533 & 0.58 & 0.14601 & 0.0082 & 0.01968 & 0.00018 & 0.35 & 126 & 1 & 138 & 7 & 363 & 109 & 126 & 1 & 8.7 \\
\hline Zircon_77_099 & 4050 & 1875 & 0.44 & 0.13597 & 0.0022 & 0.01988 & 0.00012 & 0.35 & 126.9 & 0.8 & 129 & 2 & 181 & 33 & 127 & 1 & 1.6 \\
\hline Zircon_15_024 & 392 & 273 & 0.67 & 0.16894 & 0.0051 & 0.01992 & 0.00018 & 0.29 & 127 & 1 & 158 & 4 & 675 & 58 & 127 & 1 & 19.6 \\
\hline Zircon $32 \quad 045$ & $\underline{179}$ & $\underline{100}$ & $\underline{0.53}$ & $\underline{0.1626}$ & $\underline{0.0125}$ & 0.01988 & $\underline{0.0002}$ & $\underline{0.16}$ & $\underline{127}$ & $\underline{1}$ & $\underline{153}$ & $\underline{11}$ & $\underline{579}$ & 157 & $\underline{127}$ & $\underline{1}$ & $\underline{17.0}$ \\
\hline Zircon_05_012 & 1389 & 1360 & 0.93 & 0.13917 & 0.0025 & 0.02002 & 0.00013 & 0.36 & 127.8 & $0 . \overline{\overline{8}}$ & 132 & 2 & 218 & 37 & 128 & $\overline{1}$ & 3.2 \\
\hline Zircon 73094 & 156 & $\underline{70}$ & 0.43 & $\underline{0.15866}$ & $\underline{0.0109}$ & $\underline{0.01999}$ & $\underline{0.00025}$ & $\underline{0.24}$ & $\underline{128}$ & $\underline{2}$ & $\underline{150}$ & $\underline{10}$ & $\underline{513}$ & 134 & $\underline{128}$ & $\underline{2}$ & 14.7 \\
\hline Zircon $65 \quad 084$ & $\overline{418}$ & $\underline{242}$ & $\overline{0.55}$ & 0.15274 & $\overline{0.0092}$ & 0.02041 & 0.00025 & $\overline{0.38}$ & $\underline{130}$ & $\underline{2}$ & $\overline{144}$ & $\underline{8}$ & $\overline{382}$ & 127 & 130 & $\underline{2}$ & 9.7 \\
\hline$\overline{\text { Zircon_17_027 }}$ & $\overline{874}$ & $\overline{449}$ & $\overline{0.49}$ & $\overline{0.14386}$ & 0.003 & $\overline{0.02047}$ & $\overline{0.00013}$ & $\overline{0.31}$ & 130.6 & $0 . \overline{8}$ & $\overline{136}$ & 3 & $\overline{240}$ & 43 & $\overline{131}$ & $\overline{1}$ & 4.0 \\
\hline Zircon_27_039 & 681 & 381 & 0.53 & 0.13827 & 0.0032 & 0.02053 & 0.00015 & 0.32 & 131 & 0.9 & 132 & 3 & 151 & 50 & 131 & 1 & 0.8 \\
\hline Zircon_75_096 & 631 & 594 & 0.90 & 0.15527 & 0.0047 & 0.02056 & 0.00018 & 0.3 & 131 & 1 & 147 & 4 & 426 & 61 & 131 & 1 & 10.9 \\
\hline Zircon_58_076 & 291 & 226 & 0.74 & 0.17386 & 0.007 & 0.02058 & 0.00019 & 0.23 & 131 & 1 & 163 & 6 & 681 & 86 & 131 & 1 & 19.6 \\
\hline Zircon 11020 & $\underline{246}$ & $\underline{220}$ & $\underline{0.85}$ & 0.17373 & $\underline{0.0125}$ & $\underline{0.02061}$ & 0.00024 & $\underline{0.3}$ & $\underline{131}$ & $\underline{2}$ & $\underline{163}$ & 11 & $\underline{644}$ & $\underline{129}$ & 131 & $\underline{2}$ & $\underline{19.6}$ \\
\hline$\overline{\text { Zircon_30_042 }}$ & $\overline{317}$ & $\overline{188}$ & $\overline{0.57}$ & $\overline{0.15469}$ & 0.005 & $\overline{0.02072}$ & $\overline{0.00018}$ & $0 . \overline{28}$ & $\overline{132}$ & $\overline{1}$ & $\overline{146}$ & $\overline{4}$ & $\overline{393}$ & $\overline{68}$ & $\overline{132}$ & $\overline{1}$ & $\overline{9.6}$ \\
\hline Zircon_29_041 & 212 & 155 & 0.70 & 0.18056 & 0.0065 & 0.02073 & 0.00018 & 0.24 & 132 & 1 & 169 & 6 & 730 & 73 & 132 & 1 & 21.9 \\
\hline Zircon_20_030 & 205 & 119 & 0.55 & 0.15863 & 0.0066 & 0.02064 & 0.00025 & 0.29 & 132 & 2 & 150 & 6 & 454 & 83 & 132 & 2 & 12.0 \\
\hline Zircon_76_098 & 369 & 196 & 0.51 & 0.15928 & 0.008 & 0.02065 & 0.00018 & 0.28 & 132 & 1 & 150 & 7 & 450 & 97 & 132 & 1 & 12.0 \\
\hline Zircon $59 \quad 077$ & $\underline{193}$ & 101 & $\underline{0.50}$ & $\underline{0.18072}$ & $\underline{0.0112}$ & 0.02082 & $\underline{0.00025}$ & $\underline{0.28}$ & $\underline{133}$ & $\underline{2}$ & $\underline{169}$ & $\underline{10}$ & $\underline{707}$ & $\underline{127}$ & $\underline{133}$ & $\underline{2}$ & $\underline{21.3}$ \\
\hline$\overline{\text { Zircon_35_048 }}$ & $\overline{399}$ & $\overline{295}$ & $\overline{0.70}$ & $\overline{0.17002}$ & $\overline{0.0051}$ & $\overline{0.02105}$ & $\overline{0.00027}$ & $\overline{0.44}$ & $\overline{134}$ & $\overline{2}$ & $\overline{159}$ & 4 & $\overline{555}$ & 58 & $\overline{134}$ & $\overline{2}$ & $\overline{15.7}$ \\
\hline Zircon_57_075 & 661 & 481 & 0.69 & 0.14471 & 0.0083 & 0.02101 & 0.00017 & 0.3 & 134 & 1 & 137 & 7 & 193 & 124 & 134 & 1 & 2.2 \\
\hline Zircon_52_069 & 298 & 157 & 0.50 & 0.1528 & 0.004 & 0.02117 & 0.00017 & 0.31 & 135 & 1 & 144 & 4 & 318 & 59 & 135 & 1 & 6.3 \\
\hline Zircon_71_092 & 526 & 429 & 0.78 & 0.16189 & 0.0063 & 0.02124 & 0.0002 & 0.23 & 135 & 1 & 152 & 6 & 419 & 81 & 135 & 1 & 11.2 \\
\hline Zircon_07_015 & 481 & 316 & 0.63 & 0.15395 & 0.0039 & 0.02121 & 0.00015 & 0.29 & 135.3 & 0.9 & 145 & 3 & 322 & 51 & 135 & 1 & 6.7 \\
\hline Zircon_31_044 & 788 & 602 & 0.73 & 0.15656 & 0.0033 & 0.02143 & 0.00016 & 0.35 & 137 & 1 & 148 & 3 & 331 & 44 & 137 & 1 & 7.4 \\
\hline Zircon_19_029 & 442 & 214 & 0.46 & 0.15865 & 0.0043 & 0.02141 & 0.00016 & 0.28 & 137 & 1 & 150 & 4 & 371 & 54 & 137 & 1 & 8.7 \\
\hline Zircon_06_014 & 404 & 183 & 0.43 & 0.16184 & 0.0047 & 0.02162 & 0.00018 & 0.28 & 138 & 1 & 152 & 4 & 395 & 59 & 138 & 1 & 9.2 \\
\hline Zircon_54_071 & 574 & 476 & 0.79 & 0.16803 & 0.0051 & 0.02165 & 0.00026 & 0.4 & 138 & 2 & 158 & 4 & 459 & 64 & 138 & 2 & 12.7 \\
\hline Zircon $68 \quad 088$ & $\underline{164}$ & $\underline{92}$ & $\underline{0.53}$ & $\underline{0.18947}$ & $\underline{0.0192}$ & $\underline{0.02161}$ & $\underline{0.0003}$ & $\underline{0.2}$ & $\underline{138}$ & $\underline{2}$ & $\underline{176}$ & $\underline{16}$ & $\underline{728}$ & $\underline{195}$ & $\underline{138}$ & $\underline{2}$ & $\underline{21.6}$ \\
\hline
\end{tabular}




\begin{tabular}{|c|c|c|c|c|c|c|c|c|}
\hline 074 & 160 & 97 & 0.57 & 0.20028 & 0.0078 & 0.02186 & 0.00026 & 0.31 \\
\hline Zircon $40 \quad 054$ & 445 & 456 & $\underline{0.98}$ & 0.18329 & $\underline{0.0175}$ & 0.02187 & 0.00039 & $\underline{0.38}$ \\
\hline ircon $08 \quad 016$ & $\overline{270}$ & $\overline{52}$ & $\overline{0.54}$ & 6382 & $\overline{0.0049}$ & $\overline{0.02194}$ & $\overline{00017}$ & \\
\hline Zircon_02_009 & 389 & 50 & 0.37 & 0.15864 & 0.0044 & 0.02215 & 0.00022 & ${ }^{\prime}$ \\
\hline ircon_64_083 & 498 & 54 & 0.49 & 7321 & 0.0049 & 2204 & 24 & 38 \\
\hline Zircon_22_033 & 110 & 69 & 0.60 & 0.19238 & 0.009 & 0.02212 & 0.00027 & 0.26 \\
\hline Zircon_24_035 & 172 & 95 & 0.53 & 0.16365 & 0.0064 & 0.02235 & 0.00021 & 0.24 \\
\hline ircon_50_066 & 306 & 177 & 0.55 & 0.18926 & 0.0063 & .0225 & 0.00022 & 0.29 \\
\hline Zircon $66 \quad 086$ & $\underline{245}$ & 151 & $\underline{0.59}$ & $\underline{0.16569}$ & $\underline{0.0127}$ & $\underline{0.02263}$ & $\underline{0.00024}$ & .24 \\
\hline$\overline{\text { Zircon } 33 \quad 046}$ & $\underline{66}$ & 24 & $\overline{0.35}$ & 0.22067 & $\overline{0.0125}$ & $\overline{0.02273}$ & $\overline{0.00032}$ & $\overline{0.24}$ \\
\hline$\overline{\text { Zircon_46_062 }}$ & $6 \overline{85}$ & $3 \overline{48}$ & $\overline{0.48}$ & $\overline{0.16521}$ & 0.005 & $\overline{0.02294}$ & $\overline{03}$ & $\overline{0.43}$ \\
\hline Zircon_48_064 & 139 & $\underline{59}$ & $\underline{0.40}$ & 0.19299 & $\underline{0.0113}$ & 0.02299 & $\underline{0.00}$ & 0.27 \\
\hline$\overline{\text { Zircon_74_095 }}$ & $\overline{389}$ & $2 \overline{18}$ & $\overline{0.53}$ & & & $\overline{326}$ & & $\overline{0.29}$ \\
\hline Zircon_53_070 & 306 & $\underline{141}$ & $\underline{0.44}$ & $\underline{0.20582}$ & $\underline{0.0}$ & 52 & 35 & $\underline{0.35}$ \\
\hline$\overline{\text { Zircon_04_011 }}$ & $\overline{836}$ & $\overline{117}$ & $\overline{0.13}$ & $\overline{0.16789}$ & & $\overline{991}$ & & $\overline{0.33}$ \\
\hline Zircon_78_100 & 1852 & 408 & 0.21 & 003 & & 441 & & 0.38 \\
\hline Zircon_28_040 & 227 & 12 & 0.47 & 0.18438 & 67 & 0.02439 & 24 & 0.26 \\
\hline 80_102 & 342 & 73 & 0.48 & 884 & & 42 & & 0.29 \\
\hline 6_026 & 389 & 65 & 0.40 & 326 & 0.0 & 504 & 02 & 0.29 \\
\hline Zirco & 367 & 176 & 0.46 & 886 & 54 & 251 & 19 & 0.27 \\
\hline Zircon_01_Ma-2. & 248 & 94 & 0.36 & 49 & 67 & 61 & 21 & 0.25 \\
\hline Zircon_49_065 & 193 & 95 & 0.47 & 033 & 0.0 & 331 & 83 & 0.59 \\
\hline 34_047 & 495 & 16 & 0.42 & 49 & & 68 & & 0.31 \\
\hline 7_051 & 1040 & 41 & 0.13 & 0.4161 & 0.0 & 455 & 34 & 0.39 \\
\hline -087 & 995 & 08 & 0.27 & 96 & & 25 & & 0.51 \\
\hline 082 & 973 & 123 & 0.12 & 364 & 0.0 & 11 & 72 & 0.53 \\
\hline $44 \_059$ & 949 & 61 & 0.06 & 0.77854 & 0.0 & 234 & & 0.47 \\
\hline 9_089 & 200 & 71 & 0.34 & 0.83253 & 0.0194 & 741 & & 0.51 \\
\hline -017 & 281 & 143 & 0.48 & 268 & 0.0 & 165 & 23 & 0.57 \\
\hline 080 & 321 & 80 & 0.24 & 32 & & 39 & 85 & 0.44 \\
\hline $62 \_081$ & 176 & 86 & 0.47 & 1.5908 & 0.0314 & 849 & 0.00128 & 0.41 \\
\hline Zircon_18_028 & 289 & 69 & 0.23 & 1.9703 & 0.0301 & 0.18998 & 0.00116 & 0.4 \\
\hline 42_057 & 278 & 98 & 0.34 & 1.7321 & 0.0281 & 0.16644 & 103 & 0.39 \\
\hline 1_068 & 487 & 83 & 0.16 & & & & & 0.46 \\
\hline Zircon_60_078 & 472 & 274 & 0.55 & 2.4518 & 0.0437 & 0.19444 & & 0.62 \\
\hline Zircon_13_022 & 119 & 117 & 0.93 & 4.7644 & 0.0731 & 0.31045 & 0.00196 & 0.41 \\
\hline Zircon_21_032 & 165 & 130 & 0.75 & 5.3771 & 0.0817 & 0.34567 & 0.00204 & 0.39 \\
\hline Zircon_55_072 & 367 & 155 & 0.40 & 6.9208 & 0.1003 & 0.39335 & 0.00252 & 0.44 \\
\hline
\end{tabular}

\begin{tabular}{|c|c|c|}
\hline 139 & 2 & 185 \\
\hline$\underline{139}$ & $\underline{2}$ & 171 \\
\hline$\overline{140}$ & $\overline{1}$ & $\overline{154}$ \\
\hline 141 & 1 & 150 \\
\hline 141 & 2 & 162 \\
\hline 141 & 2 & 179 \\
\hline 142 & 1 & 154 \\
\hline 143 & 1 & 176 \\
\hline 144 & $\underline{2}$ & $\underline{156}$ \\
\hline$\underline{145}$ & $\underline{\overline{2}}$ & 202 \\
\hline$\overline{146}$ & $\overline{2}$ & $\overline{155}$ \\
\hline 147 & $\underline{2}$ & $\underline{179}$ \\
\hline$\overline{148}$ & $\overline{1}$ & $\overline{160}$ \\
\hline 150 & $\underline{2}$ & $\underline{190}$ \\
\hline$\overline{152}$ & $\overline{1}$ & 158 \\
\hline 155 & 1 & 167 \\
\hline 155 & 2 & 172 \\
\hline 156 & 1 & 175 \\
\hline 159 & 1 & 171 \\
\hline 160 & 1 & 169 \\
\hline 166 & 1 & 182 \\
\hline 210 & 5 & 251 \\
\hline 300 & 2 & 307 \\
\hline 342 & 2 & 353 \\
\hline 451 & 4 & 455 \\
\hline 515 & 4 & 553 \\
\hline 569 & 4 & 585 \\
\hline 599 & 7 & 615 \\
\hline 682 & 7 & 717 \\
\hline 692 & 5 & 707 \\
\hline 948 & 7 & 967 \\
\hline 1121 & 6 & 1105 \\
\hline 992 & 6 & 1021 \\
\hline 1327 & 8 & 1271 \\
\hline 1145 & 12 & 1258 \\
\hline 1743 & 10 & 1779 \\
\hline 1914 & 10 & 1881 \\
\hline 2138 & 12 & 2101 \\
\hline
\end{tabular}

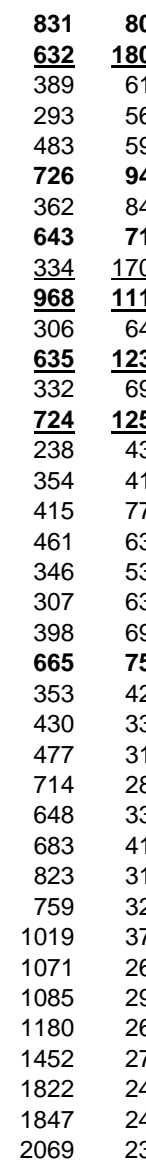

$\begin{array}{r}\mathbf{1 3 9} \\ \mathbf{1 3 9} \\ \mathbf{1 4 0} \\ \mathbf{1 4 1} \\ \mathbf{1 4 1} \\ \mathbf{1 4 1} \\ \mathbf{1 4 2} \\ \mathbf{1 4 3} \\ \mathbf{1 4 4} \\ \mathbf{1 4 5} \\ \mathbf{1 4 6} \\ \mathbf{1 4 7} \\ \mathbf{1 4 8} \\ \mathbf{1 5 0} \\ \mathbf{1 5 2} \\ \mathbf{1 5 5} \\ \mathbf{1 5 5} \\ \mathbf{1 5 6} \\ \mathbf{1 5 9} \\ \mathbf{1 6 0} \\ \mathbf{1 6 6} \\ \mathbf{2 1 0} \\ 300 \\ 342 \\ \mathbf{4 5 1} \\ \mathbf{5 1 5} \\ \mathbf{5 6 9} \\ \mathbf{5 9 9} \\ 682 \\ 692 \\ \mathbf{9 4 8} \\ 1071 \\ 1085 \\ 1180 \\ 1452 \\ 1822 \\ 1847 \\ 2069 \\ \hline\end{array}$

Note: Mineral separation was carried out using the standard methodology (crushing, sieving, density and magnetic separation, handpicking) at the mineral separation facility of the Instituto de Geologia, UNAM. Zircons were observed and imaged under cathodoluminescence, using an ELM3R luminoscope connected to a digital camera. Individual zircon ages were obtained by laser ablation inductively coupled plasma mass spectrometry (LA-ICPMS) at LEI, Centro de Geociencias, UNAM. Zircon ablation was performed with a Resolution M-50/Lambda Physik LPX220 Excimer laser, operating at a $193 \mathrm{~nm}$ wavelength and coupled to a Thermo XseriesII quadrupole ICPMS. Details of the analytic methodology can be found in Solari et al. (2010) and http://www.geociencias.unam.mx/ solari/index files/LEI/LA-ICPMS.html. Reduction of raw data was performed using an in-house developed script (Solari and Tanner, 2011), employing the Plěsovice zircon ( 337 Ma, Sláma et al., 2008) as bracketing standard. Replicate analyses of the Plěsovice zircon during the analytical sessions indicate an external reproducibility of $1.1 \%$ on the measured ${ }^{207} \mathrm{~Pb} /{ }^{206} \mathrm{~Pb}$ ratios, $0.9 \%$ on the measured ${ }^{206} \mathrm{~Pb} /{ }^{238} \mathrm{U}$ ratios, and $1.7 \%$ on the ${ }^{208} \mathrm{~Pb} /{ }^{232} \mathrm{Th}$ ratios ( $1 \sigma$ relative standard deviation). These errors are quadratically added to the quoted uncertainties for individual analyses of the analyzed zircons. The common $\mathrm{Pb}$ correction is performed employing the algebraic method of Andersen (2002), due to the impossibility to accurately measure the ${ }^{204} \mathrm{~Pb}$, which is swamped by the ${ }^{204} \mathrm{Hg}$ contained in the carrier gas. Once corrected for common $\mathrm{Pb}$, analyses that yielded $>15 \%$ or $<-5 \%$ discordant results are considered meaningless and thus discarded (values in bold in Table 2). Ages that present a porcentage of ${ }^{207} \mathrm{~Pb} /{ }^{235} \mathrm{U}$ error $>10$, and ages affected by lead loss, are not used in the concordia diagrams (underscored values in Table 2). Because the ${ }^{207} \mathrm{~Pb}$ isotope has a considerable low signal in young (i.e., $<1000 \mathrm{Ma}$ ) zircons, the mean ${ }^{206} \mathrm{~Pb} /{ }^{238} \mathrm{U}$ age is preferred to establish crystallization ages. Tera and Wasserburg (1972) concordia plots as well as error calculation were obtained using Isoplot v.3.06 (Ludwig, 2004). Sample sites coordinates are given in Table 2.

\section{REFERENCES CITED}

Andersen, T., 2002, Correction of common lead in U-Pb analyses that do not report ${ }^{204} \mathrm{~Pb}$ : Chemical Geology, v. 192, p. 59-79.

Ludwig, K.R., 2004, Isoplot/Ex: a geochronological toolkit for Microsoft Excel, Version 3: Berkeley, Berkeley Geochronology Center Publ. 4, http://www.bgc.org/isoplot_etc/software.html.

Sláma, J., Košler, J., Condon, D.J., Crowley, J.L., Gerdes, A., Hanchar, J.M., and Horstwood, M.S.A., 2008, Plešovice zircon: a new natural reference material for U-Pb and Hf isotopic microanalysis: Chemical Geology, v. 249, p. 1-35.

Solari, L.A., Gómez-Tuena, A., Bernal, J.P., Pérez-Arvizu, O., and Tanner, M., 2010, U-Pb zircon geochronology with an integrated LA-ICP-MS microanalytical workstation: achievements in precision and accuracy: Geostandards and Geoanalytical Research, v. 34, p. 5-18.

Solari, L.A., and Tanner, M., 2011, U-Pb age, a fast data reduction script for LA-ICP-MS U-Pb geochronology: Revista Mexicana de Ciencias Geológicas, v. 28, p. 83-91.

Tera, F., andWasserburg, G.J., 1972, U-Th-Pb systematics in three Apollo 14 basalts and the problem of initial Pb in lunar rocks: Earth and Planetary Science Letters, v. 14, p. 281-304. 
FIGURE A - Photographs showing details of the exposures that will be visited during the field trip.

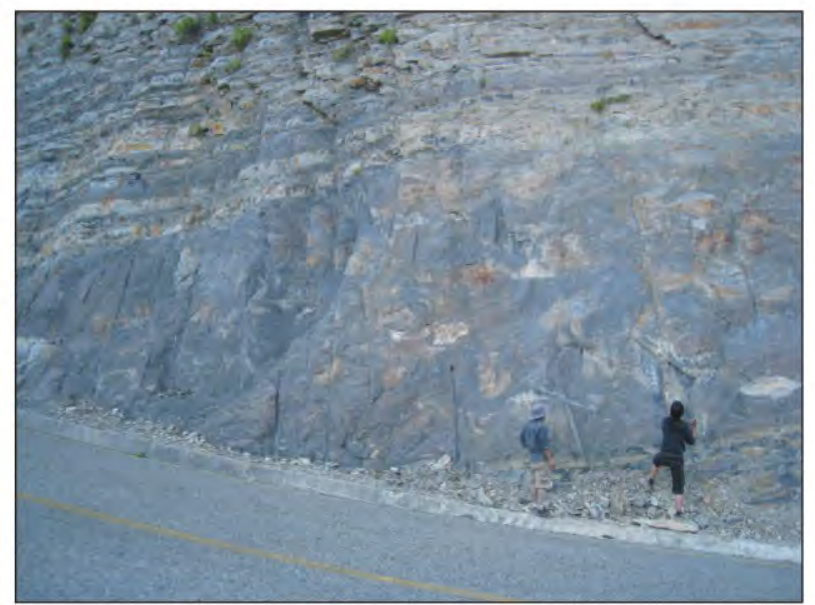

1. Volcanic flow in the Trancas Fm., Zimapán Basin. Volcaniclastic sandstone and shale interbedded with a 6-mthick felsic volcanic flow containing several lithic fragments and blocks of quartzite and limestone.

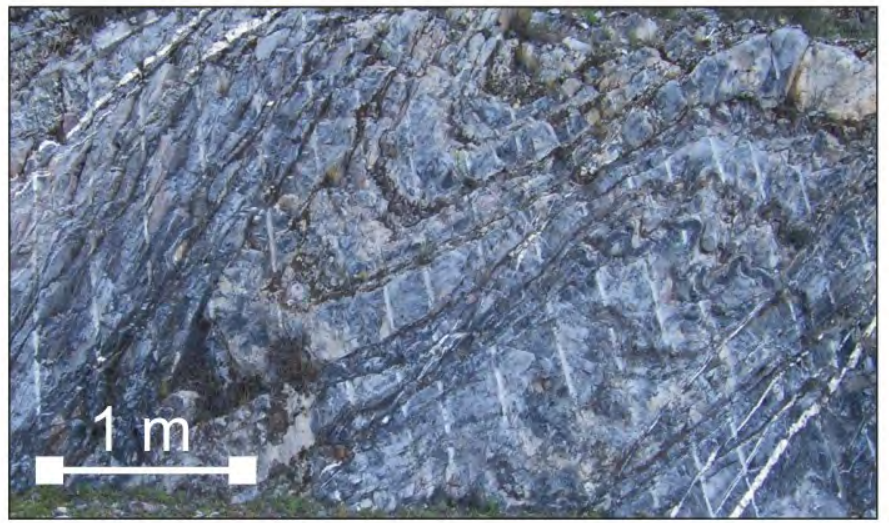

3. Train of folds in the Tamaulipas Fm., Zimapán Basin. East-vergent folds in mudstone layers interbedded with chert and thin beds of shale. Folds in the limestone layers are asymmetrical with high angle axial planes. They show angular hinges and strong variations in the layers thickness, with attenuated forelimbs and thickened hinges.

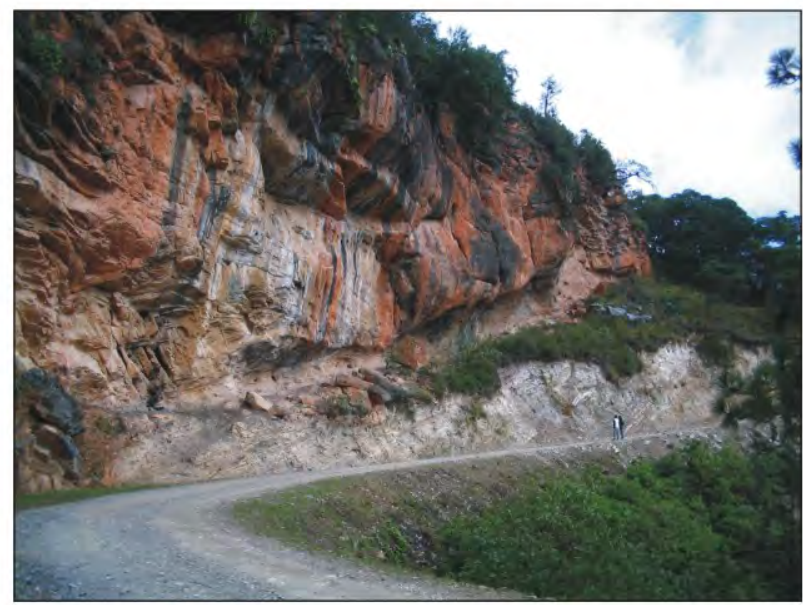

5. El Doctor Thrust. El Doctor Platform limestone thrusting to the east shale and detrital limestone of the Soyatal Fm.

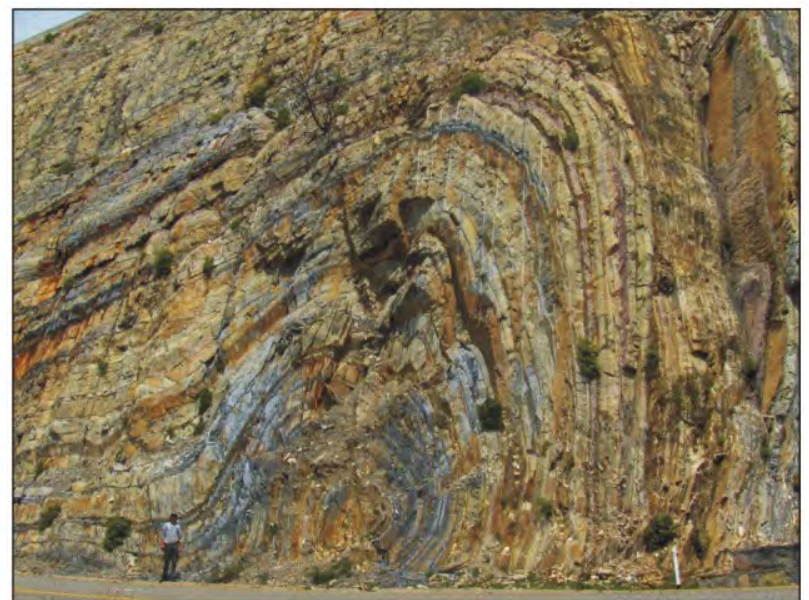

2. Deformation in the Trancas Fm., Zimapán Basin. Mesoscopic-scale asymmetrical, east-vergent anticline with an S1 pervasive axial plane cleavage. In these rocks, folds typically display rounded hinges and close to tight interlimb angles.

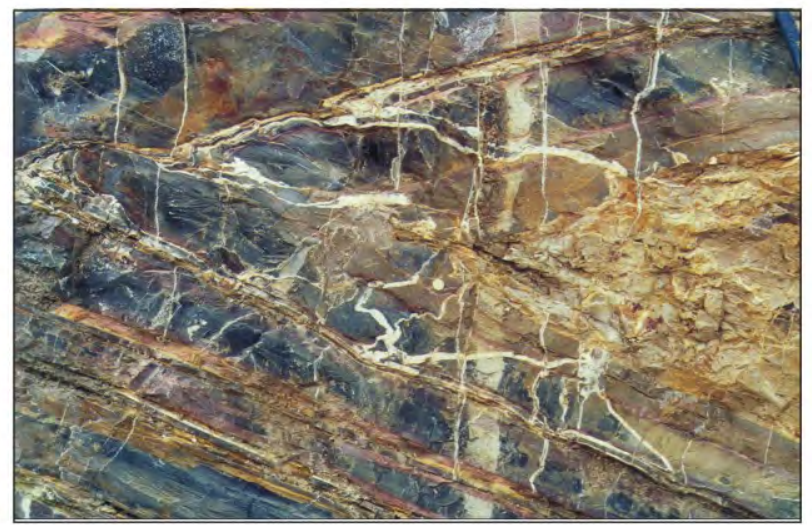

4. The icon fold in the Tamaulipas Fm., Zimapán Basin. Recumbent fold observed in the transitional contact between the Tamaulipas and Soyatal formations. The contact zone is made up of interbedded mudstone, shale, and bentonite. Folds developed in these rocks show considerable amounts of flattening, as highlighted by the tightened hinges.

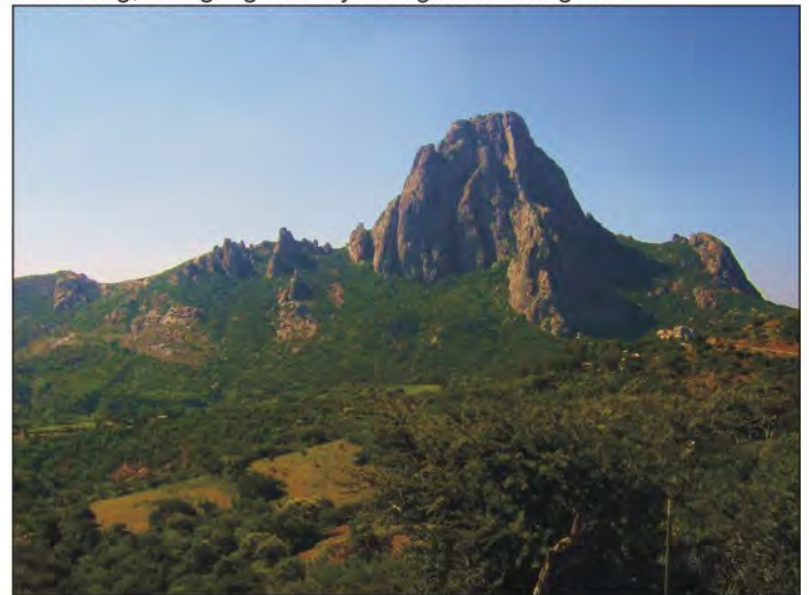

6. La Peña de Bernal. This remarkable volcanic feature was considered a volcanic neck for a long time. However, more recent studies indicate that this can be a $27 \mathrm{Ma}$ old rhyolitic dome (Aguirre-Díaz et al., 2005). 


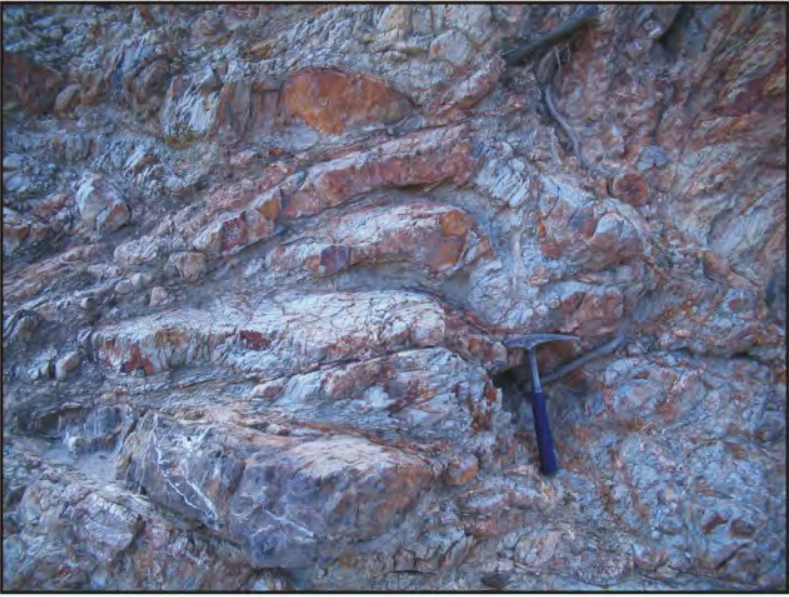

7. EI Chilar Complex. Interbedded quartz rich metasandstone and phyllite, showing a complex deformation represented by refolded folds cut by thrust faults.

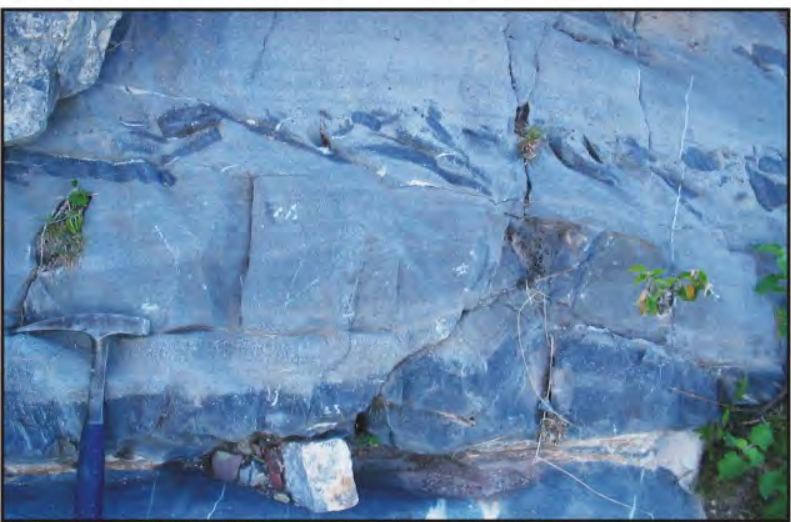

9. Peña Azul Fm. in the Tolimán area. Detrital limestone at the base of the PeñaAzul Fm., showing beds rich in chert intraclasts.

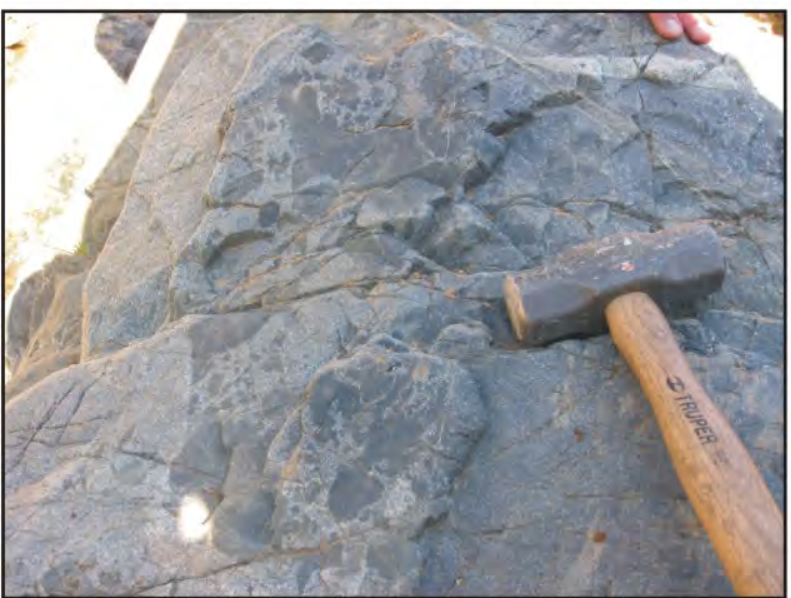

11. Tuna Manza Intrusive Complex, Esperanza area. Basaltic dike intruding and mingling with gabbro and diorite.

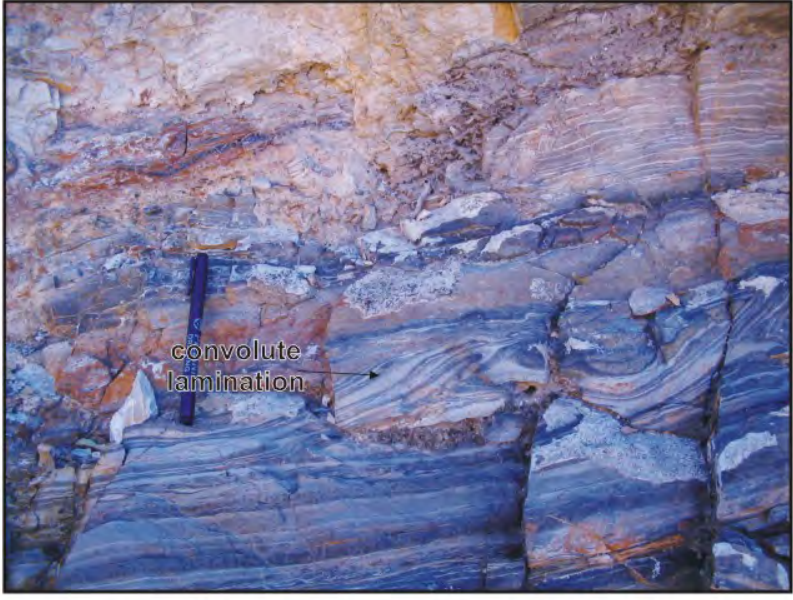

8. San Juan de la Rosa Fm., Tolimán area. Volcaniclastic turbidites showing convolute lamination.

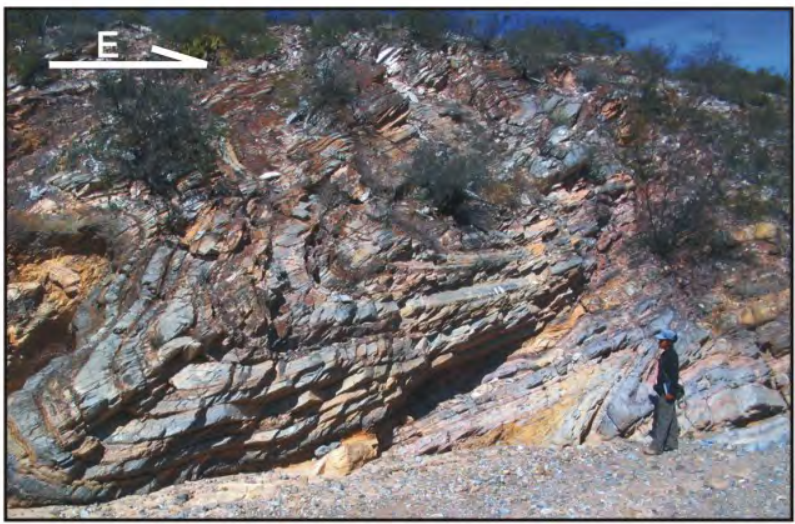

10. Folds in the Soyatal Fm. East-vergent asymmetrical inclined fold developed in detrital limestone and shale of the Soyatal Fm.

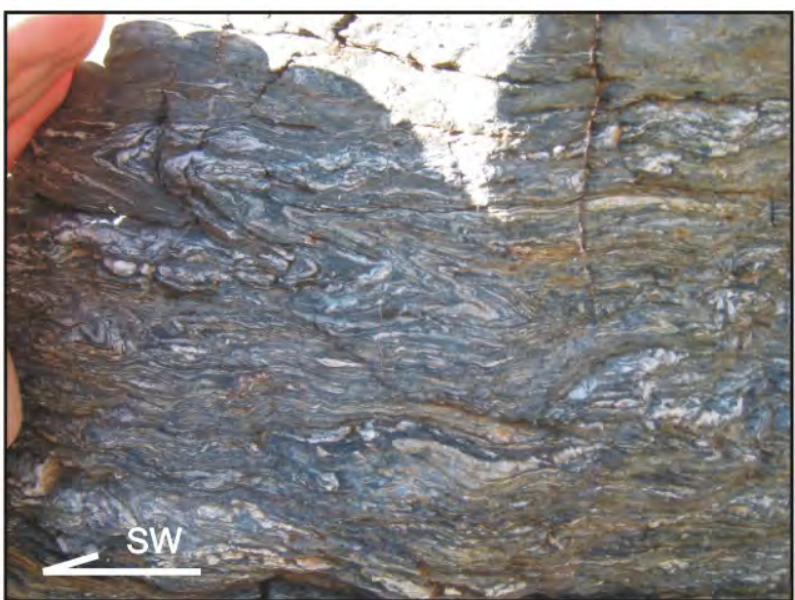

12. Santa Ana mylonitic shear zone, Esperanza area. Mylonitized metasandstone and phyllite of the Esperanza Fm. displaying asymmetrical folds showing a constant vergence to the southwest. 


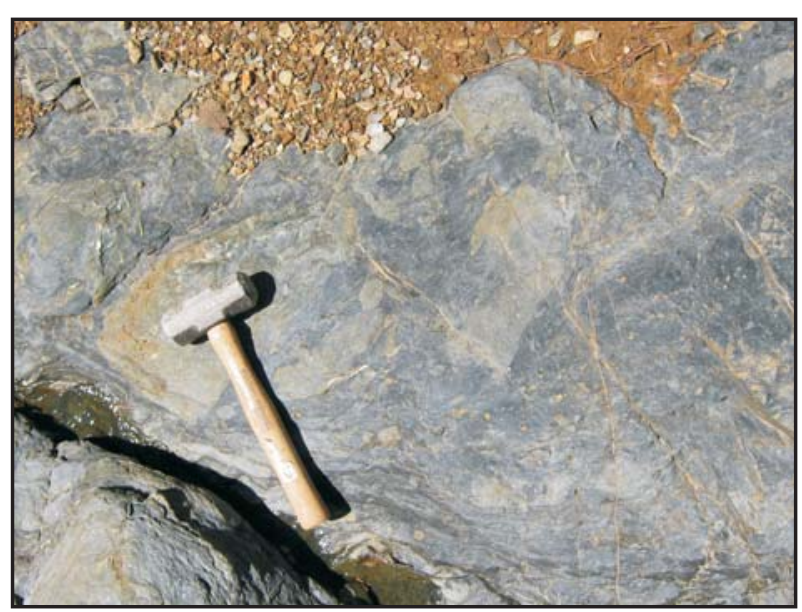

13. Esperanza Fm, Esperanza area. Peperite at the boundary of a felsic dike intruding sandstone and shale of the Esperanza $\mathrm{Fm}$.

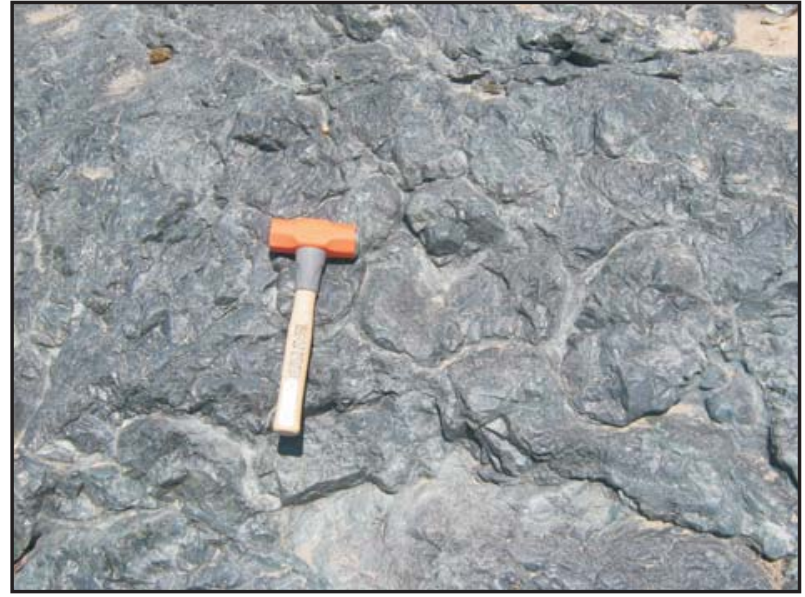

14. Arperos Fm., Arperos area. Decimeter-scale pillow basalts at the base of the Arperos Fm. 